\title{
Il mito cristianizzato di Daniela Dalla Valle all'interno dei nuovi studi sul mito
}

Daniela Mauri

\section{(2) OpenEdition}

Journals

\section{Edizione digitale}

URL: http://journals.openedition.org/studifrancesi/6165

DOI: ERREUR PDO dans/localdata/www-bin/Core/Core/Db/Db.class.php L.34 : SQLSTATE[HY000]

[2006] MySQL server has gone away

ISSN: 2421-5856

\section{Editore}

Rosenberg \& Sellier

\section{Edizione cartacea}

Data di pubblicazione: 1 novembre 2010

Paginazione: 491-496

ISSN: 0039-2944

\section{Notizia bibliografica digitale}

Daniela Mauri, «ll mito cristianizzato di Daniela Dalla Valle all'interno dei nuovi studi sul mito», Studi Francesi [Online], 162 (LIV | III) | 2010, online dal 30 novembre 2015, consultato il 07 janvier 2021. URL: http://journals.openedition.org/studifrancesi/6165 ; DOI: https://doi.org/ERREUR PDO dans / localdata/www-bin/Core/Core/Db/Db.class.php L.34 : SQLSTATE[HY000] [2006] MySQL server has gone away

\section{(c) (i) $\odot$}

Studi Francesi è distribuita con Licenza Creative Commons Attribuzione - Non commerciale - Non opere derivate 4.0 Internazionale. 


\section{DISCUSSIONI E COMUNICAZIONI}

\section{Il mito cristianizzato di Daniela Dalla Valle all'interno dei nuovi studi sul mito}

La riscrittura dei miti classici è da sempre fonte di grande interesse per gli studiosi. Vorremmo innanzitutto citare qui, sia pur brevemente e senza seguire necessariamente un criterio di carattere cronologico, alcuni volumi apparsi negli ultimi anni che ci sono sembrati particolarmente significativi e interessanti, e che trattano di varie figure mitologiche. Queste ultime, divenute poi figure letterarie, hanno attraversato i secoli producendo numerose varianti, pur rimanendo legate ad una sostanza di fondo che crea, appunto, il mito stesso. Lionello Sozzi, nel suo bel libro Amore e Psiche - un mito dall'allegoria alla parodia (Bologna, Il Mulino, 2007) cita opportunamente, "en exergue" una frase di Mircea Eliade, tratta da Aspects du mythe: «Le mythe fournit des modèles pour la conduite humaine et confère par là même signification et valeur à l'existence [...]. Connaître le mythe, c'est apprendre le secret de l'origine des choses». Sozzi analizza il mito di Amore e Psiche a partire dalla 'favola' di Apuleio e seguendo nel tempo, in un corpus di testi vastissimo, scelti soprattutto nell'ambito della letteratura francese e italiana, ma non solo, le varie interpretazioni e le modificazioni che tale mito ha subito. Il volume è completato da un apparato iconografico e da una appendice, «La metamorfosi di un mito. La favola di Amore e Psiche nelle arti figurative», ad opera di Cinzia di Cuonzo.

Michele Mastroianni, dopo aver pubblicato nel 2000 presso le Edizioni dell'Orso (Alessandria) una edizione molto accurata e ricca di apparati dell'Antigone de Sophoclès (1542) di Calvy de La Fontaine, ha dato alle stampe nel 2004 un volume importante, Le "Antigoni" sofoclee del Cinquecento francese (Firenze, Leo Olschki Editore). Mastroianni ha analizzato il mito di Antigone in cinque testi francesi che si rifanno alla tragedia di Sofocle. Si tratta dei testi di Gentien Hervet (1541), di quello sopra citato di Calvy de La Fontaine, di quello di Georges Rataller (1550 e 1570) dell'opera di Jean Lalemant (1557), di Jean-Antoine de Baï (1573) e infine di quello - forse il più interessante - di Robert Garnier (1580). Questo studio è particolarmente significativo, perché mette a punto le varianti e i temi legati alla figura mitica della figlia e sorella di Edipo, ed è accuratissimo dal punto di vista filologico. Il volume si chiude con un'appendice che tratta dell'«Antigone medievale e rinascimentale».

Vorremmo inoltre segnalare tre volumi che sono il frutto di tre convegni Internazionali di Studi svoltisi a Gargnano del Garda a Palazzo Feltrinelli e organizzati dalla sezione di Francesistica del Dipartimento di Scienze del linguaggio e Letterature straniere comparate dell'Università degli Studi di Milano. Tali convegni, che hanno luogo ogni due anni, sono denominati "Seminari Balmas - Letteratura e Immaginario, Lingua e Testo" e sono appunto dedicati alla memoria di Enea Balmas. Il primo di questi volumi è "La cruelle douceur d'Artémis". Il mito di Artemide-Diana nelle lettere francesi (Milano, Cisalpino, 2002) in cui il mito di Artemide-Diana viene studiato dall'antichità ai giorni nostri. Nelle sue considerazioni finali, Liana Nissim, che è anche la curatrice del volume, afferma, offrendoci un'altra 'definizione' di mito: «Enea Balmas [...] nous a appris que l'étude de la présence d'un mythe dans la littérature est toujours le moyen le plus avantageux pour en vérifier les valeurs invariables et en 
même temps les variations les plus symptomatiques, la permanence d'un imaginaire universel et les fluctuations infinies dont la présence désigne les changements de la vision du monde» (p. 411). Gli altri due volumi sono dedicati rispettivamente al mito di Medea e a quello di Elena di Troia, sempre nella stessa ottica di visione 'plurale' e diacronica di queste figure nell'ambito della letteratura francese. Si tratta di Magia, gelosia, vendetta. Il mito di Medea nelle lettere francesi, a cura di Liana Nissim e Alessandra Preda (Milano, Cisalpino, 2006) e di Hélène de Troie dans les lettres françaises (Milano, Cisalpino, 2008) sempre a cura delle due stesse docenti. Segnaliamo inoltre un volume della rivista «Ponts/Ponti - Langues Littératures Civilisations des Pays francophones». Si tratta del volume n. 7 della rivista (Milano, Cisalpino, 2007) che reca il titolo Présences du mythe e che indaga appunto, nella prima parte, sulla presenza di alcuni miti nell'ambito delle letterature francofone.

In questo panorama di studi dedicati appunto al mito e ai miti, intendiamo offrire particolare attenzione al bel volume di Daniela Dalla Valle, Il mito cristianizzato. Fedra / Ippolito e Edipo nel teatro francese del Seicento, Berna, Peter Lang, 2006, 260 pp., di cui forniamo qui una recensione.

Il testo di Daniela Dalla Valle è diviso in due sezioni: la prima è dedicata al mito di Fedra e Ippolito, la seconda al mito di Edipo. Entrambe le sezioni iniziano con un elenco degli autori, a partire dagli antichi sino a quelli, francesi ma non solo, del XVI e del XVII secolo che si sono occupati nelle loro opere di questi due miti. Tali elenchi - collocati rispettivamente nel I e nel VI capitolo -, in cui per ogni autore si citano i titoli e un breve riassunto dei testi che saranno poi oggetto di analisi all'interno del volume, si rivelano molto utili, perché offrono un panorama sia della fortuna che i due miti hanno incontrato nel corso dei secoli, sia delle varie modalità con cui tali miti sono stati trattati. Ciò permette all'Autrice di non soffermarsi troppo a lungo sulle fonti - peraltro abbondantemente e sempre opportunamente citate come testi di riferimento ineludibili - e di dedicare uno spazio maggiore ai testi dei drammaturghi moderni.

La sezione iniziale si apre con un capitolo, intitolato significativamente «Prima di Racine», in cui vengono analizzate le opere basate sul mito di Fedra/Ippolito e pubblicate, appunto, precedentemente al grande capolavoro raciniano, che verrà peraltro preso in esame dall'Autrice al termine di tale sezione come punto di arrivo insuperato e insuperabile. Il capitolo si apre con una analisi dell'Hippolyte (1635) di La Pinelière, sino ad ora trascurato dalla critica. Pur giudicandolo di «scarsa qualità lirica e drammatica» (p. 13), la Dalla Valle lo considera un momento importante nell'evoluzione del mito; infatti, rispetto alla fonte senecana, peraltro seguita fedelmente, l'autore francese opera alcune aggiunte apprezzabili, come la riflessione di Teseo sulla condizione dei sovrani che serve «anche ed essenzialmente a descrivere e sostenere la funzione della corte, umana e divina come ambiente della tragedia e la funzione di Teseo come protagonista della tragedia stessa» (p. 17). Ciò porta a considerare quest'opera come una "tragedia di palazzo", in cui non sono gli dei, come invece avveniva nel mito classico, a provocare gli eventi funesti, bensì gli uomini, proprio perché vivono in un ambiente di corte in cui tutto è 'opaco'. L'opera si presenta pertanto come una tragedia barocca essenzialmente umana e desacralizzata. Il capitolo prosegue con l'analisi di altri tre testi: l'Hypolite di Gabriel Gilbert (1647), l'Hippolyte di Matthieu Bidar (1675) e Phèdre et Hippolyte di Jacques Pradon (1677). La prima tragedia è legata al modello senecano probabilmente ripreso attraverso la mediazione dell'opera omonima di Robert Garnier (1573), ma inserisce su queste fonti alcuni elementi dell'Ippolito incoronato di Euripide. Tale innesto della fonte greca su quella latina si ritroverà poi nella Phèdre di Racine. Anche nel caso dell'opera di Gilbert si tenta di evitare, nello scioglimento, il problema dell'intervento divino, ricorrendo al mito, cioè alla misteriosa apparizione fantastica del mostro che uccide 
Ippolito, ripresa dalle fonti classiche. La seconda tragedia ha con queste ultime un rapporto più tenue, mentre sembra essersi ispirata più da vicino a quella di Gilbert. $\mathrm{Si}$ tratta di un testo tipicamente barocco in cui prevale il gusto per l'inganno e la finzione. La terza tragedia, infine, è stata scritta e messa in scena in concorrenza diretta con la Phèdre di Racine. Se da un lato il testo di Pradon si avvicina a quello del grande tragediografo, in quanto anche qui troviamo il personaggio di Ippolito innamorato di Aricie, dall'altro se ne distacca anche per il fatto di rappresentare Fedra come la fidanzata, e non la moglie, di Teseo: pertanto il tema dell'incesto viene a cadere. Inoltre, manca la confessione finale della protagonista, che segue Ippolito nella sua fuga. Queste tre tragedie rappresentano pertanto dei personaggi piuttosto lontani da quelli mitici e la loro storia si svolge in un ambiente più vicino alla corte del Re Sole che a quella di Teseo. Ma, soprattutto, rimane problematico e sostanzialmente abbandonato il tema del rapporto uomo/dio. Anche il mostro che provoca la morte di Ippolito viene visto da questi autori come una sorta di "deus ex machina" introdotto artificiosamente, che contrasta evidentemente con la vraisemblance.

Il terzo capitolo tratta delle «Variazioni intorno al mito di Fedra e Ippolito» e si sofferma innanzitutto su una interpretazione tragicomica del mito stesso, quella presente nell'Innocence descouverte di Jean Auvray (1609). La fonte diretta, in questo caso, che viene individuata per la prima volta dall'Autrice, è la novella inserita nel vol. X dell'Asino d'oro di Apuleio, novella in cui una matrigna è innamorata del figliastro. A questa source primaria si aggiunge comunque quella senecana. Nel 1609 il genere tragicomico è al suo inizio e Auvray tenta di utilizzare una storia tragica che ricalca quella di Fedra/Ippolito, inserendo tuttavia degli elementi di comicità come, ad esempio, la presenza di un servo parassita. Pur non riuscendo a creare una vera tragicommedia come verrà definita dal Guarini, l'autore francese tenta di rinnovare la tradizione del mito antico già rimaneggiata, peraltro, da Apuleio. L'Autrice prosegue trattando un'altra interessante variazione del mito: in alcune pièces, infatti, i personaggi di Fedra, Ippolito e Teseo vengono sostituiti rispettivamente dalle figure storiche di Fausta, Crispo e Costantino, le cui vicende familiari hanno una grande somiglianza con quelle narrate nel mito antico. Tale 'sovrapposizione' permette agli autori moderni di risolvere il problema dell'influsso divino sulla vita e sul destino degli uomini e di affermare pertanto una diversa concezione religiosa e ideologica, che accentua invece, da un lato, la funzione attiva degli uomini, e, dall'altro una visione provvidenziale. Ciò accade, soprattutto, nella tragedia latina e didattica dei Gesuiti, $\mathrm{ma}$ anche in alcuni testi italiani e francesi. L'Autrice si sofferma in particolare su due opere teatrali: quella di Grenaille, L'innocent malheureux, ou la mort de Crispe (1639), e quella di Tristan l'Hermite La mort de Chrispe ou les malheurs domestiques $d u$ Grand Constantin (1645). La prima introduce, rispetto alla vicenda storica, una figura femminile che ama di amore ricambiato Crispo, il che aprirà la strada, nelle tragedie secentesche che s'ispirano al mito di Fedra, alla rielaborazione del personaggio di Ippolito non più adepto di Diana, ma a sua volta giovane innamorato. La seconda, invece, si allontana maggiormente, rispetto alla pièce di Grenaille, dalla storia della famiglia di Costantino. Quest'ultimo, infatti, rimane al di fuori delle vicende negative che tormentano la sua famiglia e in quanto vittima innocente diviene il portavoce di una concezione provvidenziale della storia molto vicina allo spirito del teatro dei Gesuiti, anche se va sottolineato che, introducendo un personaggio nuovo, quello di Costanza, innamorata di Crispo, Tristan si avvicina al capolavoro raciniano.

Il capitolo IV intitolato «Fuori dalla Francia», si concentra sul problema, cui l'Autrice ha già fatto cenno precedentemente, della trasformazione o adattamento del mito classico, in cui la bybris umana veniva punita dagli dei 'pagani', ad una concezione cristiana in cui non è più possibile attribuire la colpa di tragiche vicende a Dio. In particolare, la Dalla Valle qui si sofferma su tre elementi: l'introduzione di 'ombre', 
personaggi morti o soprannaturali, connotate di particolari funzioni religiose; la concezione della tragedia di Fedra e Ippolito come espressione della vanitas; la trasformazione del 'mito' classico in una 'storia' - romana o medievale - «più facilmente adattabile alla dimensione cristiana» (p. 62). In questo capitolo vengono inoltre esaminate alcune opere italiane, oltre a una novella L'Hippolyte sarmate, ambientata in Polonia, dello scrittore Jean-Pierre Camus (novella peraltro riportata nell'Appendice finale del volume).

Il capitolo V, che conclude la prima parte, è tutto dedicato alla Phèdre di Racine. Tale capitolo costituisce un'analisi estremamente originale del sia pur studiatissimo testo del tragediografo francese. La Dalla Valle parte infatti dalla osservazione che nelle opere precedenti a Racine, la comparsa del mostro che provoca la morte di Ippolito «urta con la vraisemblance e con la bienséance, e soprattutto con il rifiuto classico del merveilleux» (p. 73). Nella Phèdre raciniana, invece, l'apparizione del mostro evocata nel famosissimo récit de Théramène non turba più di tanto, poiché sin dall'inizio della tragedia vengono evocati i mostri, quelli vinti da Teseo e in particolare il Minotauro. La stessa Fedra appare, e si sente, come un mostro morale che, attraverso la mediazione di Enone, calunnia Ippolito. Viene inoltre sottolineato molto acutamente dall'Autrice il fatto che qui, per la prima volta, assistiamo a una lotta tra il mostro ed Ippolito, che esce vincitore, degno erede di suo padre, benché poi il suo cavallo imbizzarrito ne provochi la morte. Inoltre, uccidendo il mostro, Ippolito è praticamente causa anche del suicidio del Mostro-Fedra.

La seconda parte che inizia, come si è detto, con l'elenco iniziale delle opere che trattano del mito di Edipo (capitolo VI), prosegue con un altro capitolo intitolato «Prima di Corneille». L'Autrice esamina qui la Thébaïde di Jean Robelin del 1584, opera probabilmente composta in polemica con Garnier che segue fedelmente i classici e adotta con rigore le unità. Robelin, invece, si disinteressa totalmente delle unità e rivela un certo gusto per l'orrore, forse di origine senecana, ma ormai già presente e accettato sulle scene francesi. La Dalla Valle precisa comunque che l'interesse per il mito di Edipo in Francia all'inizio dell'epoca moderna è stato piuttosto limitato. Quello che era difficilmente accettabile non era tanto il tema dell'incesto, che finì per divenire un tema tragico abbastanza diffuso, «quanto piuttosto la potenza del destino, del fatum, che prevale sul libero arbitrio degli uomini, talvolta sulla volontà degli dei» (p. 118). Dopo aver analizzato le fonti classiche, Sofocle e Seneca, sottolineandone le varianti fondamentali, l'Autrice si sofferma su l'Edipe di Jean Prévost (1614), che è sostanzialmente una imitazione di Seneca da cui riprende soprattutto la parte orrifica del linguaggio, ma che si allontana dall'autore latino soprattutto per quanto riguarda il tema della virtù necessaria per sconfiggere il fato e nel sottomettere quest'ultimo al potere degli dei. Tuttavia, precisa l'Autrice, una rielaborazione autonoma è solo abbozzata in Prévost. Più decise sono le varianti introdotte nei Rivaux amis di Boisrobert, di cui è difficile stabilire quale sia la fonte classica modello dello scrittore. Si tratta di una tragicommedia dall'intreccio molto complesso in cui è presente il tema dell'incesto, ma anche quello del doppio amore di un personaggio per due donne, riconducibile probabilmente alla Filli di Sciro, pastorale drammatica di Guidubaldo Bonarelli. Nel paragrafo successivo l'Autrice ritorna sull'Edipe di Prévost, analizzandone in particolare i cori. Attraverso questo esame, è possibile affermare che lo scrittore francese afferma che l'anima sopravvive alla morte, ma che, bevendo le acque del Lete, essa ritorna libera di rivivere senza coscienza del passato.

Nel capitolo VIII, vengono esaminate alcune variazioni sul mito di Edipo, e specificamente il rapporto tra $\mathrm{i}$ già citati Rivaux amis di Boisrobert e La vida es sueño di Calderón. Quest'ultimo testo si fonda sul motivo dell'«ineluttabilità delle profezie e dell'inutilità di ogni tentativo umano di sottrarvisi» (p. 145). A questo motivo si collega quello del conflitto tra la scienza dell'oroscopomanzia e il libero arbitrio. L'autore 
francese Boisrobert si serve della fonte spagnola sia per trarne una novella nel 1657, sia, appunto per scrivere Les Rivaux amis del 1639. Se infatti la fonte principale è Calderón, lo scrittore introduce alcuni elementi che ricordano da vicino l'intreccio edipico: il motivo dell'incesto, qui tra fratello e sorella, l'allontanamento del figlio che viene poi riconosciuto al termine della tragicommedia con una modalità che ricorda quello di Edipo nelle fonti classiche. Il testo di Boisrobert è quindi nel contempo 'ipertesto' dell'opera di Calderón e della storia edipica e rappresenta una correzione barocca e cattolica di quest'ultima, esaltando la virtù contro la hybris e anticipando così alcuni tratti della tragedia corneliana del 1659 .

Il capitolo IX è dedicato proprio all' Edipe di Corneille e, anche in questo caso, come accade per la Phèdre di Racine, l'Autrice riesce a fornire un'analisi ricca di nuovi spunti interessanti. Le fonti di riferimento sono, anche per Corneille, Sofocle e Seneca, ma l'autore francese ha basato l'intreccio della sua tragedia su due centri d'interesse, aggiungendo, per accontentare il gusto dell'epoca, una storia d'amore tra Dircée, figlia di Laio e Giocasta, e Thésée, re d'Atene. Nella prima parte dell'azione Edipo funge quindi principalmente da ostacolo all'amore tra i due giovani. Solo a partire dall'atto IV CEdipe diviene il vero protagonista e la vicenda 'classica' del mito si sovrappone allora alla storia d'amore creata da Corneille. Il complesso intreccio, che l'Autrice afferma, con una bella immagine, essere come la sovrapposizione di un cerchio su un'ellisse, non deve essere considerato come una giustapposizione di due vicende, ma come «una scelta innovativa nella rilettura corneliana del mito edipico» (p. 160). Inoltre, uno scrittore cristiano e allievo dei Gesuiti come Corneille non poteva accettare il mito così come era stato trasmesso dalle fonti classiche, che vedevano il destino come responsabile delle colpe degli uomini, ma doveva introdurre la dimensione cristiana del peccato. Per far ciò l'autore francese interviene proprio sul personaggio di Edipo che, da figura tirannica e machiavellica - come appare nella prima parte - si trasforma in un eroe tragico, angosciato dalla consapevolezza delle sue colpe, il parricidio e l'incesto, che si autoanalizza e cerca di sollevarsi dal destino che lo schiaccia. Tale terribile destino di Edipo renderà possibile, attraverso l'accecamento volontario, visto come miracoloso intervento provvidenziale, la salvezza della città di Tebe. Il sangue che cola dagli occhi dell'eroe provoca, infatti, la salvezza dei moribondi e rende pertanto possibile una «definizione cristologica del personaggio» (p. 172). Nel paragrafo successivo la Dalla Valle intende mettere in evidenza come l'Edipe corneliano sia stato criticato da D'Aubignac, in quanto tale confronto tra $\mathrm{i}$ due autori non è ancora stato sufficientemente analizzato. Se Corneille segue i modelli antichi, si rende conto tuttavia che essi vanno riadattati al gusto del pubblico moderno, un pubblico teatrale, e di dames, più che di savants. Piacere al pubblico diventa infatti la 'legge' cui il tragediografo francese guarda con maggiore attenzione. Proprio per questo tipo di destinatari il tragediografo inserisce, come si è visto, la storia d'amore tra Dircée e Thésée, e allontana dalla scena la catastrofe dell'accecamento di Edipo, che viene solo narrato. Tutti gli interventi adottati sulle fonti antiche porterebbero per Corneille a una maggiore vraisemblence. Tuttavia D'Aubignac critica questa tragedia proprio per una mancanza di vraisemblance e anche, in certi punti, di bienséance. Nonostante ciò, Corneille - «fortunatamente per il teatro francese» (p. 186), commenta l'Autrice - rifiuta di obbedire a una 'legislazione' troppo rigida, come già aveva fatto per il Cid.

Il capitolo X «Dopo Corneille» si occupa dell'Edipe dell'ugonotto Tallemant de Réaux, testo peraltro mai pubblicato, che sembra essere stato concepito prima della tragedia corneliana, ma in seguito corretto sulla base di quest'ultima. Riferito a una sola fonte, quella sofoclea, che viene tuttavia interpolata da alcune aggiunte che suggerirebbero, appunto, un influsso del testo di Corneille. In quanto ugonotto, tuttavia, Tallemant non incontrò gli stessi problemi del suo predecessore riguardo alla visione 
dell'umanità totalmente sottoposta al destino, non dovendo mutarla radicalmente. Per questo autore, l'umanità è incapace di capire gli dei «perché il rapporto tra i due universi è sempre doloroso, forse impossibile, e gli dei sono lontani, nascosti, separati» (p. 199).

Infine, sempre nel X capitolo, l'Autrice, dopo aver riportato il testo dell'Hyppolite Sarmate di Jean-Pierre Camus, inserita nelle sue Décades Historiques, analizza la novella che, pur essendo apparentemente diversa dalla storia mitica, tuttavia si avvicina molto ad essa, ma integrata, in alcuni passaggi, con il modello biblico. Non solo. La novella appare particolarmente interessante anche perché, per certi aspetti, «la lettura del mito di Edipo si sovrappone all'interpretazione del mito di Ippolito» (p. 212) In questo modo, «i due miti 'fatalisti' degli antichi si assestano in una lettura barocca e cristiana» (ibid.).

Il volume è completato da un'appendice contenente alcuni testi e pièces liminaires riferentesi ad alcune delle opere di cui l'Autrice ha trattato nel suo studio, e da un'ampia e aggiornata bibliografia.

Per concludere, possiamo senz'altro affermare che lo studio di Daniela Dalla Valle è uno strumento fondamentale, per l'acutezza e l'accuratezza di analisi, la vastità degli argomenti trattati (in numerosi casi, esami di testi di prima mano o ben poco studiati), per affrontare lo studio della fortuna francese, che va al di là del periodo strettamente barocco, di due dei miti più amati dalle letterature di tutti i tempi. 